\title{
Analysis of boiler water level system Based on the fuzzy control
}

\author{
Tianyi Zhang ${ }^{1}$, Fengzhi Dai ${ }^{1,2}{ }^{*}$, Peng Lu ${ }^{1}$ \\ ${ }^{1}$ Tianjin University of Science and Technology, China; \\ ${ }^{2}$ Tianjin Tianke Intelligent and Manufacture Technology CO., LTD, China \\ E-mail:*daifz@tust.edu.cn \\ www.tust.edu.cn
}

\begin{abstract}
Based on the three-stroke water supply system, this paper analyzes the performance characteristics of the boiler water level control system in reality, so as to reason out the appropriate fuzzy control rules, design fuzzy controller, and applied to the control system, so that the system for self-adjustment of PID parameters, constitute a fuzzy PID control system. On this basis, this paper analyzes the performance, advantages and characteristics of two control systems: the traditional PID control system and the fuzzy PID control system, and simulates the parameters of the input variables for comparison and analysis.
\end{abstract}

Keywords: Fuzzy PID, three-pulse, MATLAB, PID control

\section{Introduction}

Boilers are playing a very important role in factory production. The main task of boiler production is to produce and transfer heat energy and converting energy from coal or natural gas to other energy sources such as electricity. It is a large-area capacity facility used in industrial production processes. Therefore, boiler safety is an issue of great concern to us.

Boilers are often subject to a wide variety of objects and system disturbances. It would be unrealistic to rely on the operator to keep it running accurately in the plant for long periods of time. Therefore, the study of automatic control systems for boiler equipment is a general trend ${ }^{1}$.

In the boiler drum water level fuzzy control system, the core control method is the fuzzy control. Fuzzy control is a control method based on fuzzy logic theory, fuzzy set theory and fuzzy language variables ${ }^{2}$.

The fuzzy PID boiler water level control system is investigated and analyzed in terms of model and characteristics ${ }^{3,4}$. The design, simulation, and analysis were performed in the Fuzzy Logic Toolbox in MATLAB.
The fuzzy control is not necessarily an accurate mathematical analysis of the controlled object, but rather a combination of fuzzy control algorithms and PID control to control non-linearity, make the boiler level system stable and robust.

\section{System Control Principle}

Generally speaking, the larger the boiler drum capacity, the larger the volume of steam mixture and steam in the drum. Sufficient space is required for separation and circulation, and the moisture content of the generated steam is much more stringent. In turn, the water level of the cartridge is also very strict.

In a double-pulse control system, automatic adjustment of the water supply valve does not occur in time, resulting in a delay. Therefore, the water level in the packet will deviate due to the delay. The tri-pulse control system introduces the control amount of the water supply signal based on the level and steam signals of the double-pulse control system. 


\section{1. boiler water level system structure}

Boiler drum water level control system consists of Economizer, Drum Boiler, Down tube, Superheater, Lifter tube, Water Supply and so on.

The boiler obtains water through a water supply control valve, then passes through an economizer where it absorbs heat from the burning fuel, regulates it to the amount of water supply, dynamically equilibrates the steam mixture and liquid water in the steam package by regulating the water supply, then passes through the water cooling wall around the furnace in the top connection box, and finally returns to the steam package ${ }^{3}$. Boiler drum water level control system is shown in the Fig.1.

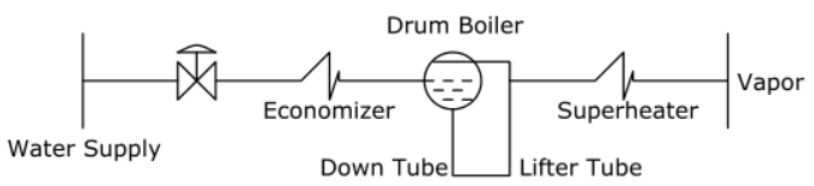

Fig.1. Boiler drum water level control system

\section{2. principles of PID control}

Start with the classical PID control, which consists of proportional, integral, and differential components. The proportional part of this is to maintain system stability . As the scale factor increases, the adjustment time becomes shorter and the resulting system deviation becomes smaller. However, if the proportionality factor is too large, oscillations can occur. Therefore, the scale factor $\mathrm{K}$ should be appropriate to achieve the effect of small transition time, small static difference, and high stability.

The function of the integral part is to eliminate systematic deviations. The larger the integration time, the weaker the system's ability to eliminate deviations and the longer the transition time. The smaller the integration time, the greater the ability to eliminate deviations that may cause the system to oscillate. The role of the microcomponent is to improve the dynamic performance of the system. Classical PID Control Schematic Block Diagram is shown in Fig.2.

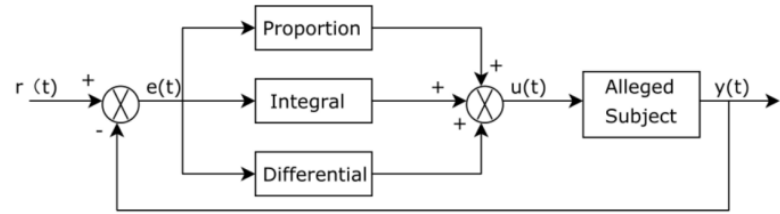

Fig.2. Classical PID Control Schematic Block Diagram

The control rule is

$$
\mathrm{U}(\mathrm{t})=K_{P}\left[\mathrm{e}(\mathrm{t})+\frac{1}{T_{i}} \int_{0}^{t} e(t) d t+T_{D} \frac{d_{e(t)}}{d_{t}}\right]
$$

When selecting a PID controller, choose a simpler and more accurate incremental PID controller.

$$
\Delta U(k)=u(k)-u(k-1)
$$

Only the first three deviations are needed to calculate $\Delta \mathrm{U}(\mathrm{k})$, and the incremental PID control algorithm is now widely used.

\section{3. cascade control systems}

Cascade systems form an inner and outer closed loop in the system configuration. The execution of the control sequence of the system is the outer ring first, followed by the inner ring. Cascade Control Systems is shown in Fig.3.

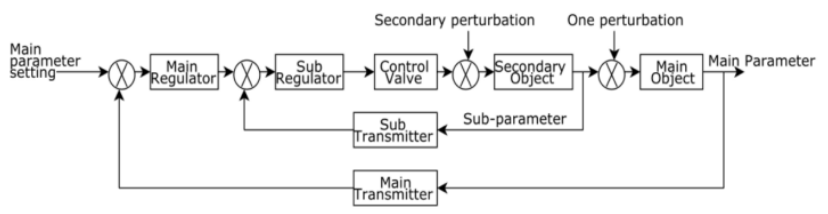

Fig.3. Cascade Control Systems

\section{Design of Fuzzy Controller}

The following introduces the basic structure of the fuzzy controller, and the fuzzy controller designed in this paper.

\section{1. the basic structure}

It consists of four parts: Knowledge Base, Fuzzy Reasoning, Fuzzification of Inputs, and Accuracy of Outputs.

First, the input quantity is fuzzified and reasoned about by the knowledge base, and finally the output quantity is decoupled and refined. Basic structure of the fuzzy controller is shown in Fig.4. 


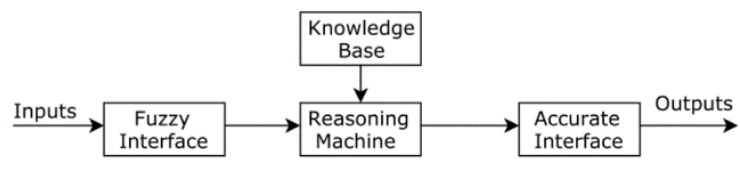

Fig.4. Basic structure of the fuzzy controller

\section{2. design process for fuzzy controller}

There are the following steps.

- Structural design with two-dimensional fuzzy controller for maximum efficiency. Controller has two inputs and three outputs.

- Fuzzing of Inputs. The set of input and output words is denoted by $\{\mathrm{NB}, \mathrm{NM}, \mathrm{NS}, \mathrm{ZO}, \mathrm{PS}, \mathrm{PM}, \mathrm{PB}\}$ and the subordinate function is chosen to be an isosceles triangle. Isosceles triangle affiliation function image is shown in Fig.5.

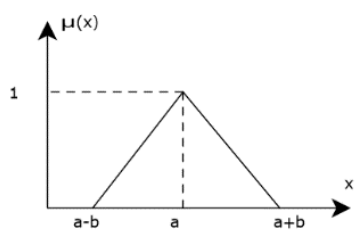

Fig.5. Isosceles Triangle Affiliation Function Image

- Define fuzzy rules. Make separate tables of the three output variables and analyze how they change on their own as E and Ec change. The 49 control rules are deduced from practical experience. The $\mathrm{Kp}$ is shown in the Table 1.

Table 1. Kp Fuzzy Rules Table

\begin{tabular}{cccccccc}
\hline elec & NB & NM & NS & ZO & PS & PM & PB \\
\hline NB & PB & PB & PM & PM & PS & PS & 0 \\
NM & PB & PB & PM & PM & PS & 0 & 0 \\
NS & PM & PM & PM & PS & 0 & NS & NM \\
ZO & PM & PS & PS & 0 & NS & NM & NM \\
PS & PS & PS & 0 & NS & NS & NM & NM \\
PM & 0 & 0 & NS & NM & NM & NM & NB \\
PB & 0 & NS & NS & NM & NM & NB & NB \\
\hline
\end{tabular}

The rule table reasoning for $\mathrm{Ki}$ and $\mathrm{Kd}$ is the same as above.

- Clarifying Variables. In order to obtain accurate output variables of the control system, the weighted average method (center of gravity method) is used to solve the fuzzy.

(C) The 2021 International Conference on Artificial Life and Robotics (ICAROB2021), January 21 to 24, 2021

\section{Control System Modeling and Simulation}

Design of automatic adjustment of steam packet level according to boiler water level dynamics.

\section{1. classical PID control modeling}

From empirical data, under unit step perturbation of feedwater flow rate, floating velocity of boiler water level $\mathrm{V}=0.05 \mathrm{~mm} / \mathrm{s}$.

The time constant $\mathrm{T} 2=15 \mathrm{~s}$, so the transfer function is determined as $\mathrm{G}(\mathrm{W})=0.05 /\left(15 \mathrm{~S}^{\wedge} 2+\mathrm{S}\right)$. Under steam flow perturbation, the amplification factor $\mathrm{K} 2=5, \mathrm{~T} 2=15 \mathrm{~s}$, yielding the transmission $\mathrm{G}(\mathrm{D})=-0.05 / \mathrm{S}+\mathrm{S} /(15 \mathrm{~S}+1)$.

The boiler water level model is built using the SIMULINK toolbox in the MATLAB environment, and the three parameters $\mathrm{Kp}=10, \mathrm{Ki}=0.0045$, and $\mathrm{Kd}=0$ are obtained by the improvised test method ${ }^{4}$. Classical PID control model is shown in Fig.6.

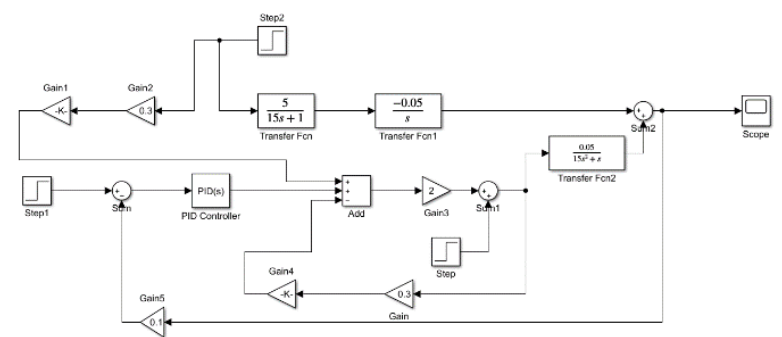

Fig.6. Classical PID control model

\section{2. fuzzy PID control modeling}

Add a fuzzy logical controller with associated fuzzy rules to the classical PID control simulation model. Fuzzy PID control model is shown in Fig.7.

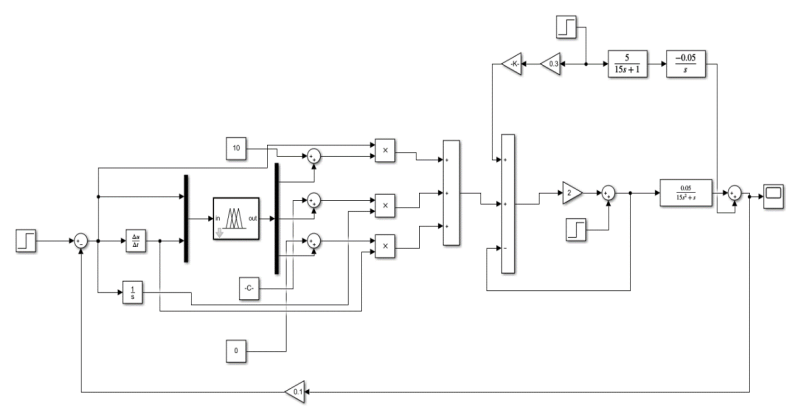

Fig.7. Fuzzy PID control model

\section{3. simulation results analysis}

- Simulation graphics with only level perturbation 
Simulation diagrams with only level disturbance is shown in Fig.8. The left figure shows the simulation results of the classical PID control model and the right figure shows the simulation results of the fuzzy PID control model.

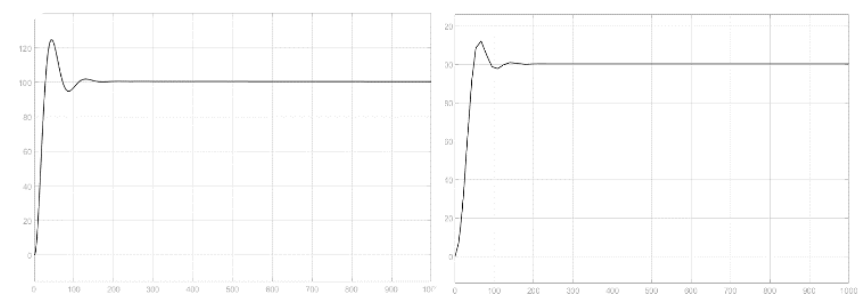

Fig.8. Simulation diagrams with only level disturbance

- Add steam disturbance at 500s in addition to level disturbance. Simulation diagrams for liquid level disturbance and vapor disturbance is shown in Fig.9.

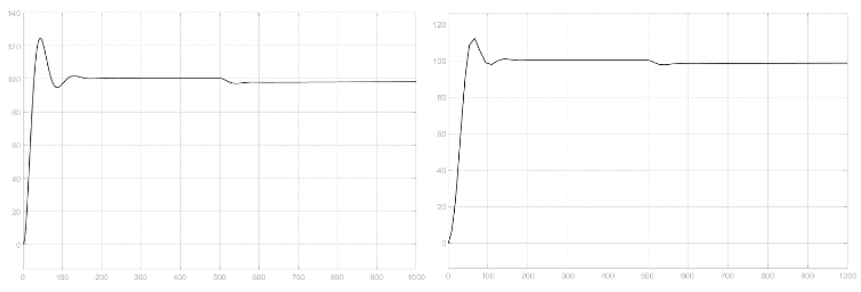

Fig.9. Simulation diagrams for liquid level disturbance and vapor disturbance

From the two graphs, the fuzzy PID control system (right) has better overshoot and setting time than the classical PID control system (left). When multiple perturbations are added, the fuzzy PID control system is more stable.

\section{Conclusion}

By analyzing the dynamic and static characteristics of the automatic drum water level control system, the paper constructed a conventional PID simulation model and a fuzzy PID simulation model to validate the results. It can be concluded that the fuzzy PID control system has a small amount of overshoot, short oscillation period and short setting time.

Fuzzy PID control system has greater flexibility and robustness, and better fault tolerance.

\section{References}

1. Zhongning Li. Design and analysis of boiler water level fuzzy control system based on MATLAB, 2008, pp. 5-7.

2. Jian Yang. Water level modeling and control strategy for large marine fuel boilers. Shanghai Jiao Tong University, 2007, pp.42-43.

3. Ping Chen. Study of fuzzy PID control of boiler drum water level, 2006, pp. 5-10.

4. Na Feng, Yao Wang. MatLab simulation study of boiler drum water level fuzzy control system. Automation and Instrumentation, 2019, 34(04): 80-84+99. 